\title{
A handheld optical fiber parallel acoustic delay line (PADL) probe for photoacoustic tomography
}

Young Cho, Cheung-Chung Chang, Mansik Jeon, Chulhong Kim, Lihong V. Wang, et al.

Young Cho, Cheung-Chung Chang, Mansik Jeon, Chulhong Kim, Lihong V. Wang, Jun Zou, "A handheld optical fiber parallel acoustic delay line (PADL) probe for photoacoustic tomography," Proc. SPIE 8943, Photons Plus Ultrasound: Imaging and Sensing 2014, 89432W (3 March 2014); doi: $10.1117 / 12.2037870$

SPIE. Event: SPIE BiOS, 2014, San Francisco, California, United States 


\title{
A Handheld Optical Fiber Parallel Acoustic Delay Line (PADL) Probe for Photoacoustic Tomography
}

Young Cho*a ${ }^{* a}$ Cheung-Chung Chang ${ }^{\mathrm{a}}$, Mansik Jeon ${ }^{\mathrm{b}, \mathrm{c}}$, Chulhong Kim ${ }^{\mathrm{b}, \mathrm{c}}$, Lihong V. Wang ${ }^{\mathrm{d}}$, and Jun $\mathrm{Zou}^{\mathrm{a}}$

${ }^{a}$ Texas A\&M University, College Station, TX 77843, USA; ${ }^{b}$ Pohang University of Science and

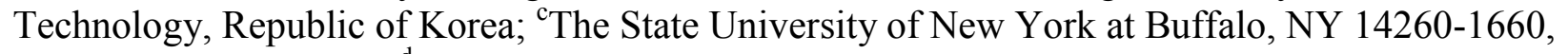
USA; ${ }^{d}$ Washington University in St. Louis, MO 63130, USA

\begin{abstract}
In current photoacoustic tomography (PAT), 1-D or 2-D ultrasound arrays and multi-channel data acquisition (DAQ) electronics are used to detect the photoacoustic signals simultaneously for "real-time" image construction. However, as the number of transducer elements and DAQ channels increase, the construction and operation of the ultrasound receiving system will become complex and costly. This situation can be addressed by using parallel acoustic delay lines (PADLs) to create true time delays in multiple PA signal channels. The time-delayed PA signals will reach the ultrasound transducer at different times and therefore can be received by one single-element transducer without mixing with each other.

In this paper, we report the development of the first miniaturized PADL probe suitable for handheld operations. Fusedsilica optical fibers with low acoustic attenuation were used to construct the 16 PADLs with specific time delays. The handheld probe structure was fabricated using precision laser-micromachining process to provide robust mechanical support and accurate alignment of the PADLs with minimal acoustic distortion and inter-channel coupling. The 16 optical-fiber PADLs were arranged to form one input port and two output ports. Photoacoustic imaging of a black-ink target embedded in an optically-scattering phantom was successfully conducted using the handheld PADL probe with two single-element transducers and two DAQ channels (equal to a channel reduction ratio of 8:1). Our results show that the PADL technique and the handheld probe could provide a promising solution for real-time PAT with significantly reduced complexity and cost of the ultrasound receiver system.
\end{abstract}

Keywords: Handheld probe, photoacoustic tomography, parallel acoustic delay line, real time

\section{INTRODUCTION}

Photoacoustic tomography (PAT) is a hybrid biomedical imaging technique which integrates rich optical absorption contrast and deep penetration in a single modality [1-3]. In PAT, upon illumination of short laser pulses, photoacoustic (PA) waves are generated by transient thermoelastic expansion following the absorption of the incident laser pulses. To receive the PA signals, two kinds of method could be used. The first one is scanning (mechanically) over multiple pointlike locations with a single-element ultrasound transducer and the second one is scanning (electronically) with an ultrasonic transducer array. Since the ultrasonic transducer array can receive multiple channels of PA signals in parallel, its imaging speed is much faster than using a single-element ultrasound transducer [4-7]. However, the imaging system is more complex and costly.

To address this issue, we have investigated a new time-delayed ultrasound receiving concept based on parallel acoustic delay lines (PADLs) [8]. By creating specific acoustic time delays using PADLs, a single-element ultrasound transducer and a one-channel data acquisition (DAQ) electronics can be used to receive multiple time-delayed PA signals in time series. As a result, the complexity and cost of ultrasound receiving systems can be significantly reduced. Low-loss fusedsilica optical fibers with different lengths were used as the PADLs to provide suitable acoustic time delays. As a result, the total length of the PADLs increased dramatically with the number of the PADLs. To avoid excessive acoustic attenuation, distortion, cross-talk, and potential mechanical fracture of the fused-silica fibers, the PADLs were laid out across a large area. This situation prevented the practical use of the PADL technique in real imaging applications.

In this paper, we report the development of a miniaturized PADL probe which is suitable for hand-held PAT operations. The PADL probe consists of 16 PADLs (made of fused-silica optical fibers) arranged into two bundles, which allows the

Photons Plus Ultrasound: Imaging and Sensing 2014, edited by Alexander A. Oraevsky, Lihong V. Wang, Proc. of SPIE Vol. 8943, 89432W · C 2014 SPIE · CCC code: 1605-7422/14/\$18 · doi: 10.1117/12.2037870 
simultaneous collection of 16 channels of the PA signals using just two single-element transducers and two channels of DAQ electronics. To minimize the size of the PADL probe, all the PADLs are tightly wound into a spiral pattern with a suitable radius of curvature to avoid excessive acoustic attenuation and distortion. To provide robust support for all the delay line structures, plastic spacers with specially designed isolation trenches were fabricated by laser micromachining. The ultrasonic transmission properties of the individual PADLs were characterized and verified using two-port ultrasound measurements. To test its PA imaging capability, we used the assembled PADL probe to collect PA signals from a black-ink target embedded in an optical phantom tissue. PAT images of the black-ink target were successfully constructed, and matched well with the actual sizes of the target.

\section{PROBE DESIGN AND CONSTRUCTION}

\subsection{PADL Design}

To construct the PADLs, fused-silica optical fibers (CeramOptec Inc., MA) with a total diameter of $\sim 220 \mu$ m were used due to their broad availability, low cost, and low acoustic attenuation at ultrasonic frequencies (i.e., $\mathrm{MHz}$ ).. Based on the acoustic properties of the optical fibers, the following design specifications were considered: (1) the time delay of the shortest PADL, and (2) the time delay increment needed to prevent overlapping of PA signals traveling in different PADLs.

The acoustic time delays (controlled by the length of the delay lines) were determined by following two criteria [8]. First, the time delay between each delay line had to be long enough to prevent signal interference from neighboring ones. The second criterion was that the PA signal in the longest $\left(8^{\text {th }}\right)$ delay line had to reach the receiving transducer before the arrival of the $1^{\text {st }}$ reflected signal ( $1^{\text {st }}$ echo in the shortest delay line). Representing $T_{1}$ (in $\mu \mathrm{s}$ ) as the time delay of the $1^{\text {st }}$ delay line, the $8^{\text {th }}$ signal will reach the receiving transducer after $T_{1}+70 \mu \mathrm{s}$ (for an incremental time delay of $10 \mu \mathrm{s}$ ), and the $1^{\text {st }}$ echo will be detected at $3 T_{1}$. Based on the second criterion, an inequality of $3 T_{1}>T_{1}+70$ is required, and therefore $T_{1}$ should be longer than $35 \mu \mathrm{s}$. The time delays and delay lengths for the 8-channel PADL array are summarized in Table 1. The sound speed of the optical fiber PADLs is around $5000 \mathrm{~m} / \mathrm{s}[9]$.

Table 1. Time delays and fiber lengths for the 8-channel PADL array.

\begin{tabular}{|c|c|c|}
\hline Fiber Number & Time Delay $(\boldsymbol{\mu s})$ & Optical Fiber Length $(\mathbf{c m})$ \\
\hline 1 & 40 & 21 \\
\hline 2 & 50 & 26 \\
\hline 3 & 60 & 31 \\
\hline 4 & 70 & 36 \\
\hline 5 & 80 & 41 \\
\hline 6 & 90 & 46 \\
\hline 7 & 100 & 51 \\
\hline 8 & 110 & 56 \\
\hline
\end{tabular}

\subsection{Fiber Spacer Design}

To securely position the optical-fiber PADLs, four pairs of spacer structures were designed (Figure 1), each having a horizontal and a vertical member assembled into a cross. On each spacer structure, there is a two-dimensional matrix of elliptical holes $(800 \mu \mathrm{m}$ long and $300 \mu \mathrm{m}$ wide) for assembling the optical fibers. Unlike circular holes, the elliptic holes can provide relief clearance in the direction of optical fiber bending, which helps to reduce the chance of breaking the optical fibers during threading. To reduce possible acoustic coupling between two adjacent optical fibers passing through the spacer structure, an "L" shaped isolation trench was cut around each elliptic hole, as seen in Figure 1a. The length and width of the isolation trenches were $2.15 \mathrm{~mm}$ and $250 \mu \mathrm{m}$, respectively. To accommodate even the longest optical fiber $(\sim 56 \mathrm{~cm})$, the horizontal spacer structure has an array of 4-by-30 elliptic holes and the vertical spacer structure has an array of 4-by-18 elliptic holes. Overall, the spacer structures measure $6.2 \mathrm{~mm}$ by $60 \mathrm{~mm}$ horizontally (x-z) and 6.2 $\mathrm{mm}$ by $35.5 \mathrm{~mm}$ vertically $(\mathrm{x}-\mathrm{y})$. These dimensions allow the longest fiber to be snugly wound with a smallest radius of curvature of around $5 \mathrm{~mm}$ without breaking. 
(a)

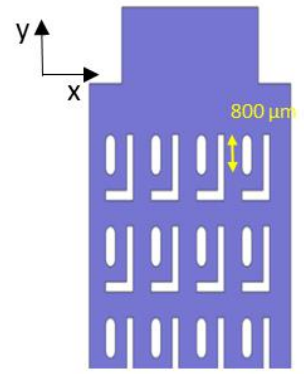

(b)

(c)

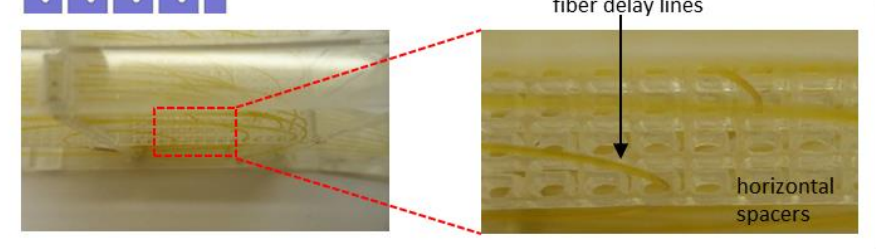

Figure 1. (a) Design of the horizontal and vertical spacers; (b) Routing PADLs through spacers in probe housing; and (c) Top and magnified views of assembled PADLs on the spacer.

\subsection{Probe Construction}

The first step in constructing the hand-held PADL probe was preparing the optical-fiber PADLs. 16 optical fibers were cut to their requisite lengths, and both ends of each optical fiber were polished. Then, from a 1.5-mm-thick acrylic sheet, a laser micromachining system (Universal Laser Systems, PLS6.75) was used to cut the input/output fiber holders, along with the top, bottom, front, and back plates of PADL housing unit, and four pairs of optical fiber spacers. The probe assembly process was conducted as follows: (1) The input and output holders for fiber arrangement were fixed on the bottom plate; (2) The front and back panels were fixed on the bottom plate; (3) The horizontal and vertical spacers were assembled crosswise. The main housing part, including the front/back panels and crossed horizontal/vertical spacers, was fixed on the bottom plate; and (4) The optical probe fibers were carefully threaded through the input port, front panel, vertical/horizontal spacers, back panel, and output ports. Since the coiled optical fibers are flexible springs under compression, the lengths of the input and output port were carefully adjusted to provide the best contact condition. Perspective and side views of the fully assembled PADL probe are shown in Figure 2.

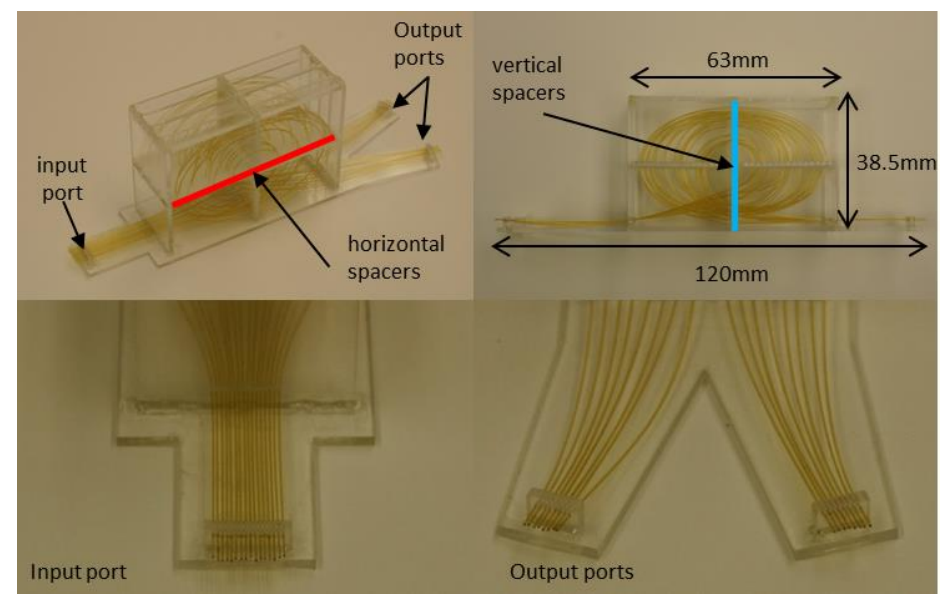

Figure 2. Fully assembled probe. 


\section{PAT IMAGING}

\subsection{PAT Imaging Setup}

The experimental setup of the PA imaging experiment is shown in Figure 3. The light source was a frequency-doubled Q-switched Nd:YAG laser (Continuum, SL2-10) with a pulse duration of $5 \mathrm{~ns}$, a pulse repetition rate of $10 \mathrm{~Hz}$, and a wavelength of $532 \mathrm{~nm}$. After it passed through several prisms, the light beam was cylindrically focused on a phantom via a cylindrical lens, parallel to the alignment of the delay lines. The laser pulse energy was less than $10 \mathrm{~mJ} / \mathrm{cm}^{2}$, much lower than the ANSI safety limit $\left(20 \mathrm{~mJ} / \mathrm{cm}^{2}\right.$ at $\left.532 \mathrm{~nm}\right)$.

The induced PA waves propagated from the phantom and travelled along the PADLs into two DAQ channels. In each DAQ channel, 8 time-delayed PA signals were sequentially detected by a single-element unfocused ultrasound transducer (Olympus NDT, V303, 1-MHz-center-frequency). The delivered PA signals were amplified by an ultrasound pulser/receiver (Olympus NDT, 5072PR). An oscilloscope (Tektronix, TDS5054) recorded these signals at a sampling rate of $5 \mathrm{MHz}$.

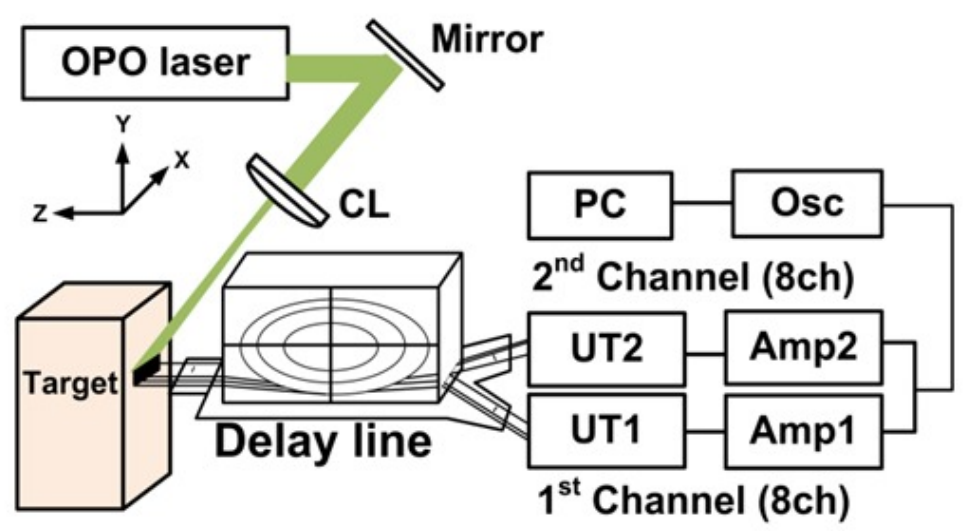

Figure 3. PAT imaging setup using the 16-channel PADL probe.

\subsection{Data Acquisition and Image Reconstruction}

The PA signals were averaged 10 times to generate one PA image. Thus, the imaging speed was $1 \mathrm{~Hz}$. Each delay line was considered as a single transducer element in an ultrasonic transducer array. Each PA signal sequentially detected by one DAQ channel was reshaped into eight separate PA signals, based on predefined time positions, measured by the ultrasound transmission experiment. The differences in acoustic attenuation in each fiber were compensated for $(0.2$ $\mathrm{dB} / \mathrm{cm}$ ). Then, a commonly used delay-and-sum beam forming method was applied to reconstruct the PA images. Briefly, the PA images were reconstructed by summing all channel data after compensating for time delays at each fiber, based on its length and matching phase. In a two-dimensional beam field along the $\mathrm{x}$ and $\mathrm{z}$ directions, a time delay $\tau_{n}$ for the $n^{\text {th }}$ PADL located on $\left(x_{n}, z_{n}\right)$ can be calculated as follows:

$$
\tau_{n}=\frac{\sqrt{\left(x-x_{n}\right)^{2}+\left(z-z_{n}\right)^{2}}-R}{c}
$$

where $R$ is the distance from a focusing point to the center of the delay line and $c$ is the speed of sound in soft tissues, $1540 \mathrm{~m} / \mathrm{s}$. Envelope detection was applied by using Hilbert transformation along the axial direction (i.e., the $z$ direction). 


\section{RESULTS AND DISCUSSION}

To demonstrate the PA imaging capability of the handheld PADL probe, we imaged an optically absorptive target ( 2.5 $\mathrm{mm} \times 1 \mathrm{~mm} \times 2.5 \mathrm{~mm}$ along the $\mathrm{X}, \mathrm{Y}$, and $\mathrm{Z}$ axes, respectively) embedded in an optically scattering tissue phantom (100 $\mathrm{mm} \times 100 \mathrm{~mm} \times 50 \mathrm{~mm}$ along the $\mathrm{X}, \mathrm{Y}$, and $\mathrm{Z}$ axes, respectively) (Figure 4a). The phantom consisted of $10 \%$ gelatin by weight and $1 \%$ intra-lipid by volume, and its reduced scattering coefficient was $\sim 9 \mathrm{~cm}^{-1}$. The object was located $2.5 \mathrm{~mm}$ below the phantom's surface. The optical absorption coefficient of the target was $\sim 100 \mathrm{~cm}^{-1}$. The raw A-line data acquired by both channels are shown in Figure $4 \mathrm{~b}$ and $4 \mathrm{c}$.

The A-line PA data from both channels were rearranged into one raw PA B-scan image, shown in Figure 5a. The reconstructed PA images are presented without and with compensation for the ultrasound attenuation $(0.2 \mathrm{~dB} / \mathrm{cm})$ in each fiber, as shown in Figure $5 \mathrm{~b}$ and $\mathrm{c}$, respectively. The reconstructed PA images match well with the photograph shown in Figure $4 \mathrm{a}$. The image contrast, defined as $\left(P A_{\text {target }}-P A_{\text {background }}\right) / P A_{\text {background }}$, is calculated to be $\sim 2.4$. The spatial resolution, defined as the one-way distance between $10 \%$ and $90 \%$ of the maximum over the minimum, is $\sim 1.1 \mathrm{~mm}$. Compared to our previous results [7], we found that the PA signals were broadened and more noisy due to the increased contact junctions and bends of the optical fibers in the small housing unit. Thus, the image contrast and spatial resolution were slightly diminished, by $20 \%$ and $37 \%$, respectively. Further, the enhanced PA image can be presented after the signal threshold of $40 \%$ (Figure $5 \mathrm{~d}$ ).

(a)
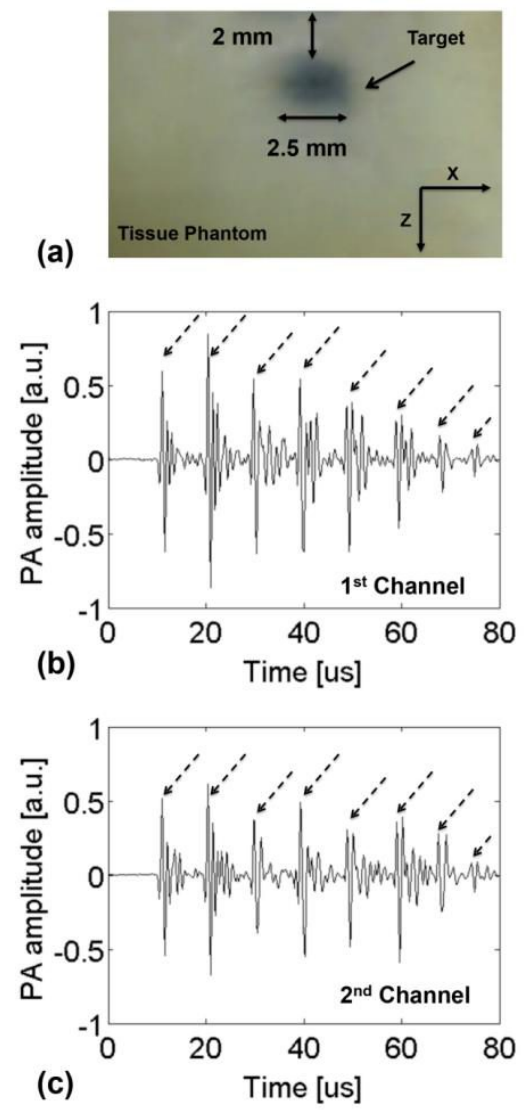

Figure 4. (a) Photograph of an optically absorbing target embedded in an optically scattering medium; (b) Raw A-line PA signals obtained by the ultrasound transducer (UT 1); and (c) Raw A-line PA signals obtained by the ultrasound transducer (UT 2). 

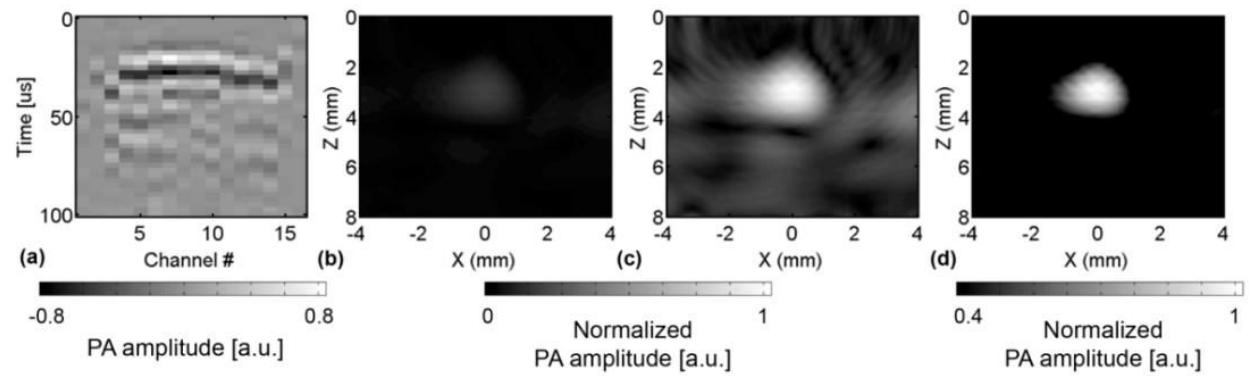

Figure 5. (a) Rearranged raw data acquired by 16 optical fibers. Reconstructed PA images without (b) and with (c) compensation for the acoustic attenuation in each fiber; and (d) threshold (40\%) PA image of (c).

\section{CONCLUSION}

In this work, we have successfully demonstrated a hand-held PAT probe using optical-fiber PADLs. To minimize their layout space, laser-micromachined spacer structures were used to wind the optical-fiber PADLs into spiral coils with suitable radii of curvature. Thus all 16 optical-fiber PADLs fit into a compact probe structure suitable for handheld operation. By properly designing and constructing the micro spacer structures and carefully threading the optical fibers, we achieved robust and accurate placement of the optical-fiber PADLs with minimal acoustic distortion and interchannel coupling. Using the hand-held PADL probe, 16 channels of PA signals can be unambiguously received with two single-element transducers and two DAQ channels, which corresponds to a channel reduction ratio of $8: 1$. The handheld PADL probe could be used to conduct real-time PAT without a complex ultrasound receiver system. It should be noted that using optical fibers as the PADLs involves intensive manual assembly. In addition, the mechanical bending of the optical fibers and the multi-point contact with the supporting structure could create unwanted mode conversion and acoustic coupling, causing distortion in the transmitted PA signals. In the future, new PADL materials, designs and construction methods will be investigated to address this issue and also to improve the PA imaging capability (e.g., a larger number of channels and higher channel reduction ration).

Acknowledgement: This work was supported in part by a grant (CMMI-1131758) from the National Science Foundation to J.Z, a grant (U54-CA136398) from the National Institutes of Health to L.V. W, and by MSIP (Ministry of Science, ICT and Future Planning), Korea, under the "IT Consilience Creative Program" (NIPA-2013-H0203-13-1001) supervised by the NIPA (National IT Industry Promotion Agency) and NRF grant of Korea government (MSIP) (2011 0030075) to C.K.

\section{REFERENCES}

[1] Oraevsky, A.A. and A.A. Karabutov, [Biomedical photonics handbook], CRC PM 125 (2003).

[2] Kim, C., C. Favazza, and L.V. Wang, "In vivo photoacoustic tomography of chemicals: high-resolution functional and molecular optical imaging at new depths, "Chem Rev 110(5), 2756-82 (2010).

[3] Wang, L., [Photoacoustic imaging and spectroscopy], CRC 144 (2009).

[4] Fronheiser, M.P., et al., "Real-time optoacoustic monitoring and three-dimensional mapping of a human arm vasculature, "Journal of biomedical optics 15(2), 021305-021305-7 (2010).

[5] Gamelin, J., et al., "A real-time photoacoustic tomography system for small animals, " Optics express 17(13), 1048910498 (2009).

[6] Song, L., et al., "High-speed dynamic 3D photoacoustic imaging of sentinel lymph node in a murine model using an ultrasound array," Medical physics 36(8), 3724 (2009).

[7] Kim, C., et al., "Handheld array-based photoacoustic probe for guiding needle biopsy of sentinel lymph nodes," Journal of biomedical optics 15(4), 046010-046010-4 (2010).

[8] Yapici, M.K., et al., "Parallel acoustic delay lines for photoacoustic tomography," Journal of biomedical optics 17(11), 116019-116019 (2012).

[9] Cheeke, J.D.N., [Fundamentals and applications of ultrasonic waves], CRC press (2012). 